\title{
Monitoreo de la remoción biológica de nitrógeno en efluentes de tenerías usando un reactor por carga secuencial
}

\author{
Monitoring of Biological Nitrogen Removal in Tannery Wastewater Using a \\ Sequencing Batch Reactor
}

\author{
Carrasquero-Ferrer Sedolfo José \\ Departamento de Ingeniería Sanitaria y Ambiental \\ Facultad de Ingeniería, Universidad del Zulia, Venezuela \\ Correo:scarrasquero@fing.luz.edu.ve \\ Pire-Sierra María Carolina \\ Universidad Centroccidental Lisandro Alvarado \\ Decanato de Agronomía Barquisimeto, Venezuela \\ Correo:carol_pire@yahoo.es
}

\author{
Rincón-Lizardo Nancy Coromoto \\ Departamento de Ingeniería Sanitaria y Ambiental \\ Facultad de Ingeniería, Universidad del Zulia, Venezuela \\ Correo:nrincon1@gmail.com \\ Díaz-Montiel Altamira Rosa \\ Departamento de Ingeniería Sanitaria y Ambiental \\ Facultad de Ingeniería, Universidad del Zulia, Venezuela \\ Correo:adiaz@fing.luz.edu.ve
}

Información del artículo: recibido: febrero de 2013, aceptado: abril de 2013

\section{Resumen}

El objetivo de esta investigación fue relacionar el proceso de remoción biológica de nitrógeno en efluente de tenerías con los perfiles de oxígeno disuelto, $\mathrm{pH}$ y potencial de óxido reducción usando un reactor por carga secuencial (SBR, Sequencing Batch Reactor) con un volumen de trabajo de 2 L. El reactor trabajó con dos secuencias operacionales, anóxica-aerobiaanóxica $(\mathrm{Ax} / \mathrm{Ae} / \mathrm{Ax})$ y aerobia-anóxica $(\mathrm{Ae} / \mathrm{Ax})$, combinadas con dos tiempos de retención celular (TRC) (15 y 25 días), y un tiempo de ciclo operacional (TCO) de 11 horas. Los perfiles se obtuvieron midiendo cada 15 minutos los parámetros: potencial de hidrógeno $(\mathrm{pH})$, oxígeno disuelto (OD), potencial de óxido reducción (ORP) y cada hora los parámetros: alcalinidad total (AT), demanda química de oxígeno total $\left(\mathrm{DQO}_{\mathrm{T}}\right)$, demanda química de oxígeno soluble $\left(\mathrm{DQO}_{\mathrm{s}}\right)$, nitrógeno total Kjeldahl (NTK), nitritos $\left(\mathrm{NO}_{2}^{-}\right)$, nitratos $\left(\mathrm{NO}_{3}^{-}\right)$y nitrógeno amoniacal $\left(\mathrm{N}^{-} \mathrm{NH}_{4}^{+}\right)$durante el ciclo operacional. La AT y el ORP fueron excelentes indicadores del proceso de remoción biológica de nitrógeno. Por el contrario, el pH no pudo utilizarse como parámetro de control debido a la capacidad de amortiguación de los efluentes de tenerías. Finalmente, esta investigación demostró que los valores de la AT y el ORP pueden usarse como parámetros de control en línea para monitorear la evolución de la remoción de nitrógeno en efluentes de tenerías (nitrificación y desnitrificación).

\section{Descriptores:}

- remoción biológica de nutrientes

- reactor por carga secuencial

- efluentes de tenería

- nitrificación

- desnitrificación 


\begin{abstract}
The objective of this research was to relate the biological nitrogen removal in tannery wastewater with profiles of $\mathrm{pH}$, alkalinity and redox potential (ORP) using a sequencing batch reactor (SBR) with a working volume of 2 L. The reactor worked under two operational sequences: anoxic-aerobic-anoxic (Ax/Ae/Ax) and aerobicanoxic (Ae/Ax), which were combined with two cell retention times (CRT) (15 and 25 days), with an operation cycle time (OCT) of 11 hours. The profiles were performed by measuring each 15 minutes the following parameters: $p H$, dissolved oxygen (DO), ORP, and each hour the parameters: total alkalinity, total chemical oxygen demand $\left(D Q O_{T}\right)$, soluble chemical oxygen demand $\left(D Q O_{S}\right)$, total Kjeldahl nitrogen (TKN), nitrite $\left(\mathrm{NO}_{2}^{-}\right)$, nitrate $\left(\mathrm{NO}_{3}^{-}\right)$and ammonia nitrogen $\left(\mathrm{N}-\mathrm{NH}_{4}^{+}\right)$. Alkalinity and ORP profile were excellent indicators of the processes of biological nitrogen removal. However, $p H$ could not be used as a control parameter, due to the buffering capacity of tannery wastewater. Finally, this research work showed that alkalinity and ORP values can be used as on-line control parameters to monitor the evolution of the nitrogen removal in tannery wastewater (nitrification and denitrification processes).
\end{abstract}

Keywords:

- nutrient biological removal

- sequencing batch reactors

- tanneries effluents

- nitrification

- denitrification

\section{Introducción}

Los reactores por carga secuencial (SBR) se utilizaron originalmente para la remoción de materia orgánica en las aguas residuales (Carucci et al., 1997). Las estrictas regulaciones establecidas para las concentraciones de las formas de nitrógeno en las descargas a los cuerpos de agua requirieron modificaciones en los sistemas SBR para lograr la ocurrencia de la nitrificación, desnitrificación y eliminación de los compuestos orgánicos (Kargi y Uygur, 2003). Estos sistemas de lodos activados no convencionales utilizan ciclos de llenado y descarga, donde la homogenización de caudales, la reacción y la sedimentación se logran en un solo reactor (EPA, 1999).

Cuando se requiere la eliminación biológica de nitrógeno, el ciclo de reacción se ajusta alternando fases aerobias y anóxicas (Mace y Mata, 2002). En la fase aerobia ocurre la remoción de la mayor proporción de carbono orgánico y el proceso de nitrificación, mientras que la fase anóxica es principalmente para la desnitrificación de los iones nitratos presentes en las aguas residuales o que se producen durante la fase aerobia, con la eliminación simultánea de los compuestos de carbono orgánico.

Los reactores por carga secuencial se han utilizado con éxito en el tratamiento de efluentes agroindustriales (Durai y Rajassiman, 2011). Estos sistemas han demostrado ser efectivos en el tratamiento de efluentes de tenerías, los cuales poseen una alta carga orgánica, elevados niveles de nitrógeno orgánico y amoniacal y compuestos inorgánicos específicos, de una manera más económica y generando una menor cantidad de subproductos que los sistemas biológicos convencionales (Farabegoli et al., 2004, Vidal et al., 2004; Ganesh et al., 2006).

Los sistemas modernos de tratamientos de aguas residuales requieren versatilidad, además de lograr altas remociones en las concentraciones de materia orgánica y nitrógeno (Han et al., 2007). El uso de los valores del potencial de óxido-reducción (ORP), potencial de hidrógeno $(\mathrm{pH})$, oxígeno disuelto (OD) y alcalinidad total (AT) como herramientas de control, representa una solución prometedora para la operación de los procesos que ocurren en el SBR, especialmente relacionándolos con las concentraciones de los nutrientes, para mejorar el control en su eliminación que hasta ahora se considera difícil debido a que en línea la medición de las concentraciones de los mismos es a menudo costosa (Akin y Ugurlu, 2005; Tanwar et al. 2008).

En los últimos años, se han llevado a cabo numerosos estudios para aplicar el monitoreo, automatización y control en plantas de tratamiento de aguas residuales, tanto en procesos continuos como por carga. En los sistemas de tratamiento por carga, los perfiles de OD; pH y ORP se utilizan para la optimización en la longitud de las fases en el ciclo operacional (Spagni et al., 2001; Spagni et al., 2007; Li et al., 2007)

El objetivo de esta investigación fue relacionar el proceso de remoción de nitrógeno en un reactor por carga secuencial a escala laboratorio durante el tratamiento de aguas residuales de tenerías con los perfiles de oxígeno disuelto, $\mathrm{pH}$, potencial de óxido reducción y alcalinidad total. 


\section{Materiales y métodos}

Los estudios se llevaron a cabo utilizando aguas residuales de una tenería ubicada en el Estado Lara, Venezuela. La empresa actualmente funciona de forma discontinua, por lo que genera tres tipos de efluentes, el efluente de pelambre $(\mathrm{P})$, el efluente que proviene del proceso de curtido (C) y el efluente del proceso de teñido $(\mathrm{T})$, que descargan en una laguna artificial ubicada a doscientos cincuenta metros de las instalaciones de la tenería.

La recolección del efluente se realizó de acuerdo con los patrones establecidos en el Método Estándar, usando el método 1060 Recolección de muestras y preservación (APHA-AWWA-WEF, 1998). La captación del agua residual se realizó de manera manual a través de un muestreo aleatorio simple, tomando las aguas residuales de la empresa en la laguna, que funciona como tanque de homogenización de los efluentes, en recipientes de polietileno de alta densidad con capacidad de 20-25 L, de color oscuro y limpios.

Para el tratamiento del efluente de la tenería se utilizó un reactor cilíndrico de vidrio de carga secuencial (SBR) de $14.5 \mathrm{~cm}$ de diámetro y $26 \mathrm{~cm}$ de alto con un volumen total del dispositivo de $4 \mathrm{~L}$. El volumen total de reacción fue de $2 \mathrm{~L}$, el volumen que ocupó el lodo activado sedimentado tras la fase de sedimentación correspondió a $30 \%$ del volumen útil del reactor, es decir, $0.6 \mathrm{~L}$, mientras que el volumen de agua residual industrial que se añadió en la fase de llenado de cada ciclo, correspondió a $70 \%$, es decir, $1.4 \mathrm{~L}$. Todo el efluente clarificado se extraía del reactor durante la fase de descarga.

El reactor funcionó de manera automatizada para la carga y descarga del agua a tratar mediante un sistema de bombeo con bombas peristálticas (Cole-Parmer, modelos 77202-60 y 77201-60) y dispositivos reguladores de tiempo (Excelline, modelo GTC-E-120AS). El reactor por carga secuencial también disponía de sistemas automatizados de agitación mecánica y suministro de aire (figura 1).

El sistema de agitación mecánica estuvo compuesto de un motor monofásico (General Electric, modelo WR60X165) de $15 \mathrm{~W}$ de potencia y $1300 \mathrm{rpm}$ ensamblado a un eje que disponía de un aspa de cuatro alas que se sumergió dentro del agua residual y del lodo (licor mezcla).

El aire se suministró a través de un compresor (SeaStar, modelo HX-308-20). El compresor de aire estaba conectado a una tubería flexible transparente de 5 $\mathrm{mm}$ enlazada en serie con un dispositivo difusor dispuesto en el fondo del reactor de $45 \mathrm{~cm}$ de largo y $4 \mathrm{~mm}$ de diámetro.

El tratamiento del efluente constó de cuatro etapas: alimentación, reacción, sedimentación y descarga. Durante el estudio se trabajó con dos secuencias operacionales: secuencia 1, anóxica-aerobia-anóxica (Ax/Ae/Ax) y secuencia 2, aerobia-anóxica (Ae/Ax), un tiempo de ciclo operacional (TCO) de 11 horas, dos tiempos de retención celular (TRC) y un tiempo de retención hidráulica (TRH) de 15,6 h. Esto dio origen a cuatro combinaciones de tratamientos (tabla 1).

La entrada del afluente se realizó desde un tanque de alimentación de vidrio de $13 \mathrm{~cm}$ de diámetro y $18,5 \mathrm{~cm}$ de altura, a través de una tubería flexible (Masterflex 06409-147) de $6 \mathrm{~mm}$ de diámetro conectada al reactor. La carga y descarga del afluente se realizó empleando dos bombas peristálticas de un solo sentido de flujo, las cuales se mantenían a un caudal constante de $93 \mathrm{ml} / \mathrm{min}$. La descarga y la extracción del licor mezcla se efectuaron a través de tuberías flexible (Masterflex 06409-147) de 6 $\mathrm{mm}$ de diámetro conectadas al reactor.

Para todos los tratamientos el tiempo de las fases de llenado, sedimentación y descarga permaneció cons-

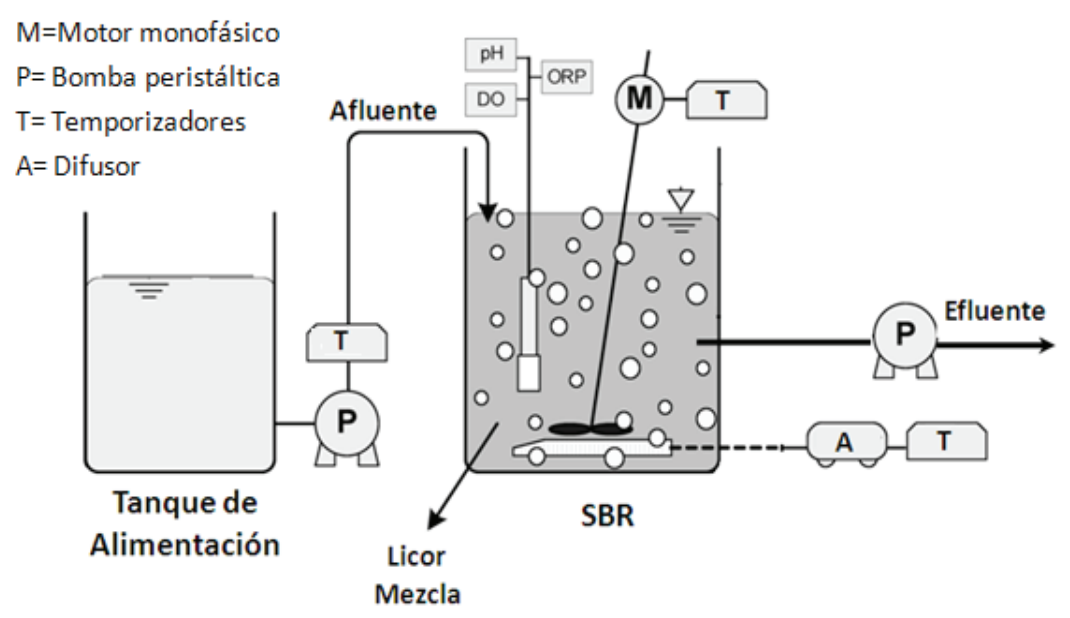

Figura 1. Descripción ilustrada del reactor por carga secuencial (SBR) 


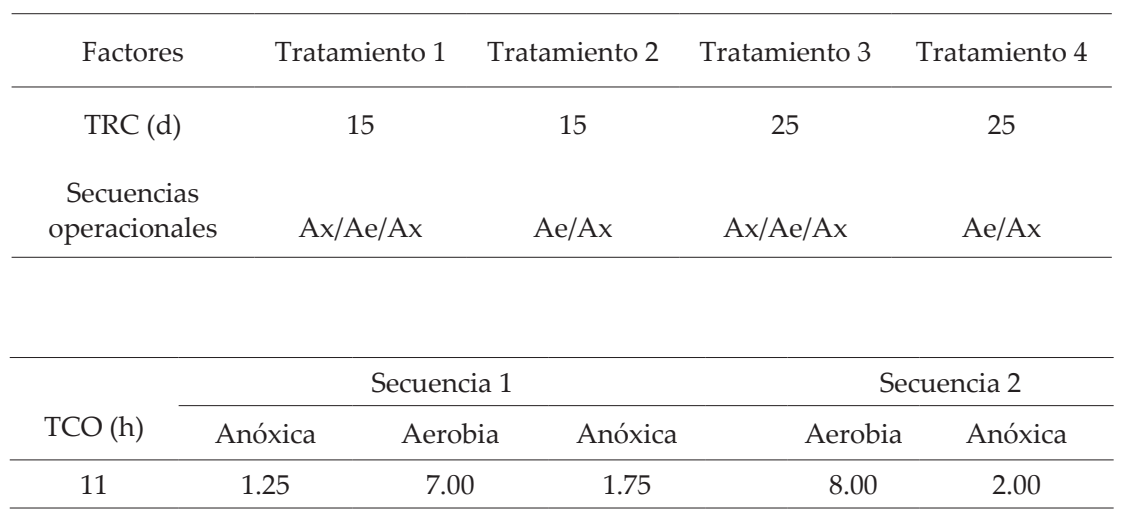

Tabla 1. Tratamientos del efluente de la tenería
Tabla 2. Duración de las secuencias operacionales de los tratamientos tante $(0.25 ; 0.50$ y 0.25 h, respectivamente). La duración de cada una de las etapas utilizadas en las secuencias operacionales se muestra en la tabla 2.

Las muestras fueron tomadas al inicio, durante y al final de cada tratamiento evaluado. Las variables evaluadas fueron $\mathrm{pH}$ (4500-B), alcalinidad total (2320-B), $\mathrm{DQO}_{\mathrm{T}}$ y $\mathrm{DQO}_{\mathrm{s}}(5220-\mathrm{C}), \mathrm{NTK}\left(4500-\mathrm{N}_{\mathrm{org}}-\mathrm{A}\right), \mathrm{N}^{-\mathrm{NH}_{4}}{ }^{+}$

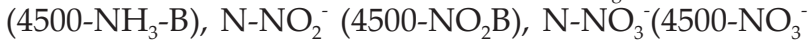
-D), PT (4500-P-C), cromo total (SM 3111 B), OD (4500O-G) según los procedimientos establecidos en el Método Estándar (APHA-AWWA-WEF, 1998). Para la determinación de la $\mathrm{DQO}_{\mathrm{S}}$, la muestra fue filtrada por una membrana estéril de éster-celulosa con un tamaño de poro de 0,45 micras. El ORP fue medido a través del método electrométrico.

Cada tratamiento fue evaluado durante un mes, realizando como mínimo nueve repeticiones por tratamiento, tres por semana, dejando la primera semana de cada mes para la estabilización del reactor debido al cambio de las condiciones operacionales entre tratamientos.

\section{Resultados y discusión}

Caracterización fisicoquímica del efluente

La caracterización fisicoquímica del efluente industrial de la tenería se presenta en la tabla 3, así como los límites establecidos por la normativa venezolana para la descarga a cuerpos de agua, contemplados en el Decreto 883 (Gaceta Oficial de la República de Venezuela, 1995). En la tabla se muestra el valor medio de cada uno de los parámetros fisicoquímicos medidos y su dispersión expresada a través de la desviación estándar.

Las características fisicoquímicas del efluente durante el periodo de muestreo mostraron gran variabilidad, reflejada en los altos valores de desviación estándar obtenidos, esto puede deberse a que la tenería tiene un funcionamiento discontinuo, con descargas intermiten- tes de las fracciones residuales en la laguna de homogeneización de los efluentes y captación de las muestras

La caracterización de las aguas residuales de las tenerías varía considerablemente en función del tamaño de la curtiembre, los productos químicos utilizados para el proceso, la cantidad de agua utilizada y el tipo de producto final elaborado por la curtiembre (Durai y Rajasimman, 2011). Sin embargo, los valores de DQO presentados en esta caracterización se encuentran cercanos al rango trabajado por autores como Orhon et al. (2000), Lefebvre et al. (2005), Szpyrkowicz y Kaul (2004) y Sivaprakasam et al. (2008), quienes reportaron una $\mathrm{DQO}_{\mathrm{T}}$ inicial de 2200, 2155, 2320 y 2426 mg/L en aguas residuales de tenerías, respectivamente. La presencia de materia orgánica en estas aguas residuales industriales se debe a la presencia de compuestos orgánicos complejos que se utilizan y generan durante la limpieza que se hace a las pieles.

Se observó que los efluentes de tenerías son ricos en nitrógeno pero pobres en fósforo. La relación $\mathrm{DQO} / \mathrm{N}$ $\mathrm{NH}_{4}^{+} / \mathrm{PT}$ fue de 100:7.40:0.70. El bajo contenido de fósforo es característico en las aguas residuales de las tenerías, tal como lo han reportado Karahan et al. (2008); Lefebvre et al. (2005); Leta et al. (2004) y DiIaconi et al. (2002).

El efluente industrial en estudio presentó valores de pH que oscilaron entre 7.2 y 8.8 unidades y se caracterizó por presentar altos valores de alcalinidad total que oscilaron entre 7500 y $4798 \mathrm{mg} \mathrm{CaCO}_{3} / \mathrm{L}$.

Con respecto a los valores de sólidos suspendidos totales y volátiles del efluente, la relación promedio de SSV/SST obtenida fue de 0.24; lo que indica la presencia de numerosas partículas inorgánicas en el efluente industrial. Los efluentes de las tenerías poseen altas concentraciones de sólidos suspendidos totales, producto de las operaciones que se llevan a cabo en este proceso productivo, en especial de la operación de pelambre, la etapa de limpieza de las pieles (Vidal et al., 2004; Lefebvre et al., 2006). 


\begin{tabular}{|c|c|c|c|}
\hline Parámetro & Unidad de expresión & Valor (media $\pm \mathrm{SD}$ ) & $\begin{array}{l}\text { Límites establecidos por } \\
\text { la norma de descarga } \\
\text { a los cuerpos de agua }\end{array}$ \\
\hline $\mathrm{pH}$ & --- & $8,0 \pm 0,8$ & $6-9$ \\
\hline Alcalinidad total & $\mathrm{mg} / \mathrm{L} \mathrm{CaCO}_{3}$ & $6148 \pm 1351$ & --- \\
\hline $\mathrm{DQO}_{\mathrm{T}}$ & $\mathrm{mg} / \mathrm{L}$ & $1810 \pm 372$ & 350 mg/L \\
\hline $\mathrm{DQO}_{\mathrm{S}}$ & $\mathrm{mg} / \mathrm{L}$ & $922 \pm 164$ & --- \\
\hline NTK & $\mathrm{mg} / \mathrm{L}$ & $211 \pm 149$ & --- \\
\hline $\mathrm{N}-\mathrm{NH}_{4}^{+}$ & $\mathrm{mg} / \mathrm{L}$ & $145 \pm 56$ & --- \\
\hline $\mathrm{N}^{-\mathrm{NO}_{2}}{ }_{2}^{-}$ & $\mathrm{mg} / \mathrm{L}$ & $28,3 \pm 14,1$ & $\mathrm{~N}-\mathrm{NO}_{2}{ }^{-+} \mathrm{N}-\mathrm{NO}_{3}-=10 \mathrm{mg} / \mathrm{L}$ \\
\hline $\mathrm{N}-\mathrm{NO}_{3}^{-}$ & $\mathrm{mg} / \mathrm{L}$ & $31,4 \pm 25,7$ & $\mathrm{~N}-\mathrm{NO}_{2}{ }^{-+} \mathrm{N}-\mathrm{NO}_{3}^{-}=10 \mathrm{mg} / \mathrm{L}$ \\
\hline NT & $\mathrm{mg} / \mathrm{L}$ & $402 \pm 183$ & $40 \mathrm{mg} / \mathrm{L}$ \\
\hline PT & $\mathrm{mg} / \mathrm{L}$ & $7,7 \pm 5,6$ & $10 \mathrm{mg} / \mathrm{L}$ \\
\hline SST & $\mathrm{mg} / \mathrm{L}$ & $3587 \pm 217$ & $80 \mathrm{mg} / \mathrm{L}$ \\
\hline SSV & $\mathrm{mg} / \mathrm{L}$ & $863 \pm 57$ & --- \\
\hline Cromo total & $\mathrm{mg} / \mathrm{L}$ & $2,3 \pm 0,0$ & $2 \mathrm{mg} / \mathrm{L}$ \\
\hline
\end{tabular}

Tabla 3. Características fisicoquímicas del efluente industrial de la tenería y límites establecidos por la normativa venezolana para la descarga a cuerpos de agua $\mathrm{n}$ : número de mediciones realizadas, $\mathrm{n} \geq 15$. $\mathrm{SD}$ : desviación estándar

\section{Perfiles de DQOT y DQOS en el ciclo operacional del reactor}

La variación de la $\mathrm{DQO}_{\mathrm{T}}$ en el ciclo del reactor por carga secuencial fue estudiada, los resultados mostraron que para los cuatro tratamientos aplicados la $\mathrm{DQO}_{\mathrm{T}}$ disminuyó durante el progreso del ciclo dentro del SBR (figuras 2 y 3), alcanzándose a detectar un valor constante de la DQO bajo el tiempo de retención hidráulica estudiado $(11 \mathrm{~h})$ para los tratamientos T2 y T4 (619 y $605 \mathrm{mg} / \mathrm{L}$, respectivamente) que pudiera representar la fracción de la DQO inerte al tratamiento biológico, tal como reportó Caruccí et al. (1999), quienes obtuvieron una DQO que permaneció remanente en el agua proveniente de una tenería luego de ser tratada en un SBR, que osciló entre 350 y $400 \mathrm{mg} / \mathrm{L}$.

En este orden de ideas, Dilaconi et al. (2002), encontraron una DQO residual de $280 \mathrm{mg} / \mathrm{L}$, que permaneció constante durante las últimas seis horas del ciclo operacional del SBR, que tenía una duración del ciclo operacional de 8 horas. Para los tratamientos 1 y 3 , en los cuales se utilizó una fase anóxica previa a la etapa aerobia con una duración de 1.25 h, se obtuvo un consumo de materia orgánica de 6 y 13\% durante esta fase. Se observó también un consumo de materia orgánica medida como $\mathrm{DQO}_{\mathrm{T}}$ en la fase anóxica posterior a la fase aerobia para todos los tratamientos, este consumo estuvo entre 12 y $20 \%$ para los tratamientos T1 y T3; y 2 y $6 \%$ para los tratamientos T2 y T4.

Hermida et al. (2000), determinaron las formas de la $\mathrm{DQO}_{\mathrm{T}}$ en los efluentes de una tenería, obteniendo que solo $16 \%$ de la $\mathrm{DQO}_{\mathrm{T}}$ inicial en estas aguas residuales es fácilmente biodegradable, mientras que $50 \%$ es rápidamente y lentamente hidrolizable, $25 \%$ es soluble inerte y $9 \%$ es particulada inerte. Las fracciones lentamente hidrolizables son absorbidas en los lodos y solubilizadas por un proceso de hidrólisis a sustratos fácilmente biodegradables, en este caso, la hidrólisis es una etapa más lenta que el crecimiento heterótrofo, por lo tanto, es el paso controlante del proceso (Orhon y Ubay,1997; Orhon et al.,1999a)

Otros autores como Karahan et al. (2008) en investigaciones sobre fraccionamiento de la DQO, obtuvieron que la DQO fácilmente biodegradable corresponde a $7 \%$ de la $\mathrm{DQO}_{\mathrm{T}}, 20 \%$ es fácilmente hidrolizable y $13 \%$ es soluble inerte. De allí se supone que el reactor por carga secuencial logra remover en primera instancia la DQO soluble conformada por la DQO rápidamente biodegradable y rápidamente hidrolizable, y posiblemente parte de la fracción lentamente hidrolizable.

La variación de la $\mathrm{DQO}_{\mathrm{S}}$ en el ciclo del reactor por carga secuencial fue estudiada, los resultados obtenidos muestran que para los cuatro tratamientos aplicados al igual que la $\mathrm{DQO}_{\mathrm{T}}$ la $\mathrm{DQO}_{\mathrm{S}}$ tuvo una tendencia constante de disminuir durante el progreso del ciclo dentro del SBR hasta a mediados de la fase anóxica donde la curva se estabilizó en valores de 240; 277; 243 y $283 \mathrm{mg} / \mathrm{L}$ para los tratamientos T1, T2, T3 y T4, respectivamente (figuras 4 y 5). Estos valores en los cuales se estabilizan las curvas de consumo de DQOs pueden corresponder a los valores de DQO soluble inerte que no se pueden remover por tratamientos biológicos. Or- 


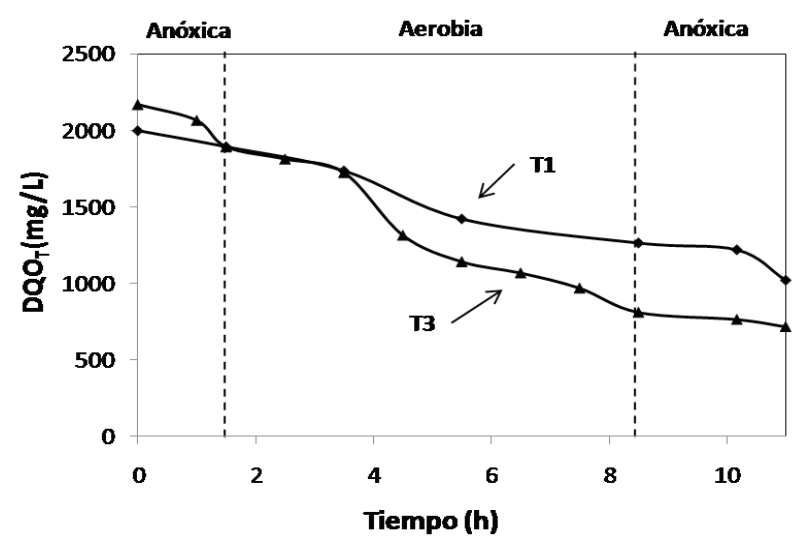

Figura 2. Evolución de la $\mathrm{DQO}_{\mathrm{T}}$ durante un ciclo de operación del SBR para los tratamientos T1 y T3

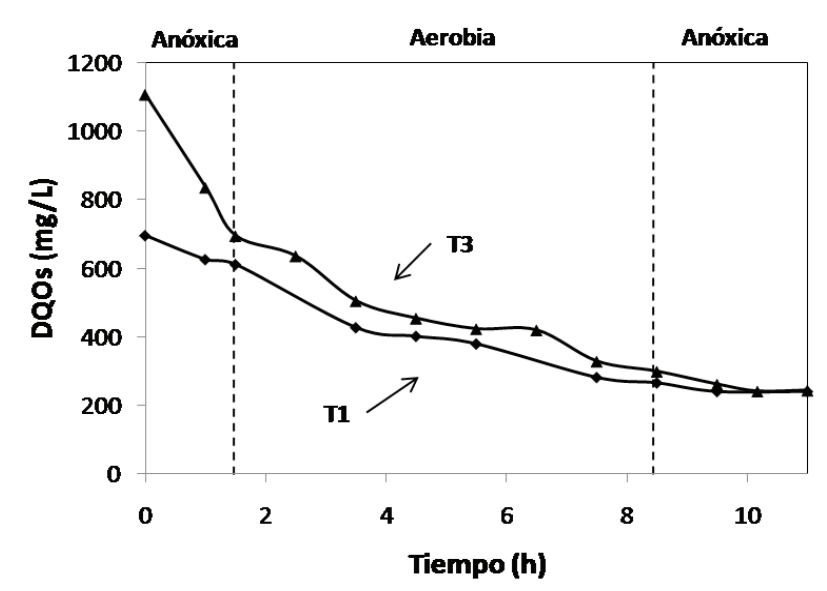

Figura 4. Evolución de la $\mathrm{DQO}_{\mathrm{S}}$ durante un ciclo de operación del SBR para los tratamientos T1 y T3

hon et al., 1999b, obtuvieron que la fracción soluble inerte oscila entre 9 y $14 \%$ para aguas de tenerías, mientras que Hermida et al. (2000) obtuvieron que esa fracción representa $34 \%$ de la $\mathrm{DQO}_{\mathrm{T}}$.

\section{Perfiles de las diferentes formas nitrógeno en el ciclo operacional del reactor}

Los perfiles de las concentraciones de las diferentes especies de nitrógeno $\mathrm{NTK}, \mathrm{N}-\mathrm{NH}_{4}{ }^{+}, \mathrm{N}-\mathrm{NO}_{3}{ }_{3}^{-} \mathrm{N}-\mathrm{NO}_{2}{ }_{2}^{-}$y las variables de control operacional, $\mathrm{pH}, \mathrm{OD}, \mathrm{ORP}$ y alcalinidad total se presentan en las figuras 6, 7, 8 y 9 durante el tiempo de ciclo operacional de $11 \mathrm{~h}$ del SBR para los cuatro tratamientos aplicados.

Las fases de cada ciclo se pueden identificar claramente de acuerdo a los perfiles de ORP y OD. Bajo con-

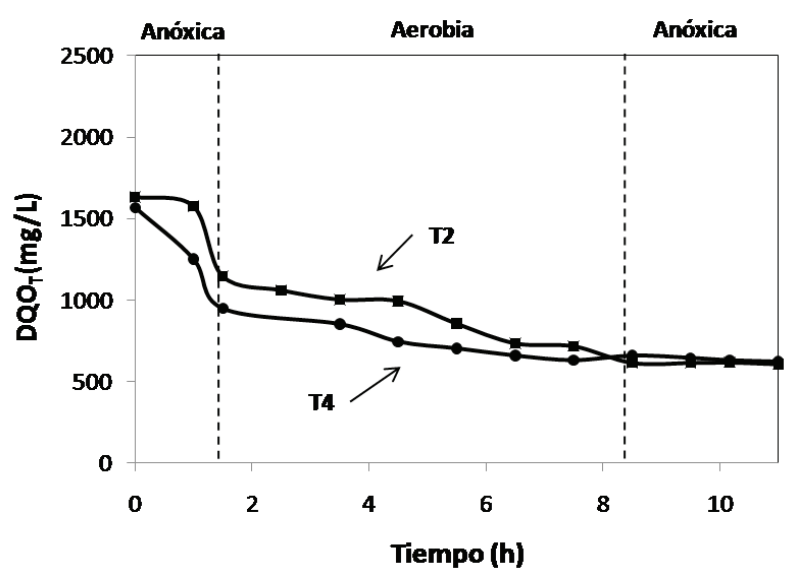

Figura 3. Evolución de la $\mathrm{DQO}_{\mathrm{T}}$ durante un ciclo de operación del SBR para los tratamientos T2 y T4

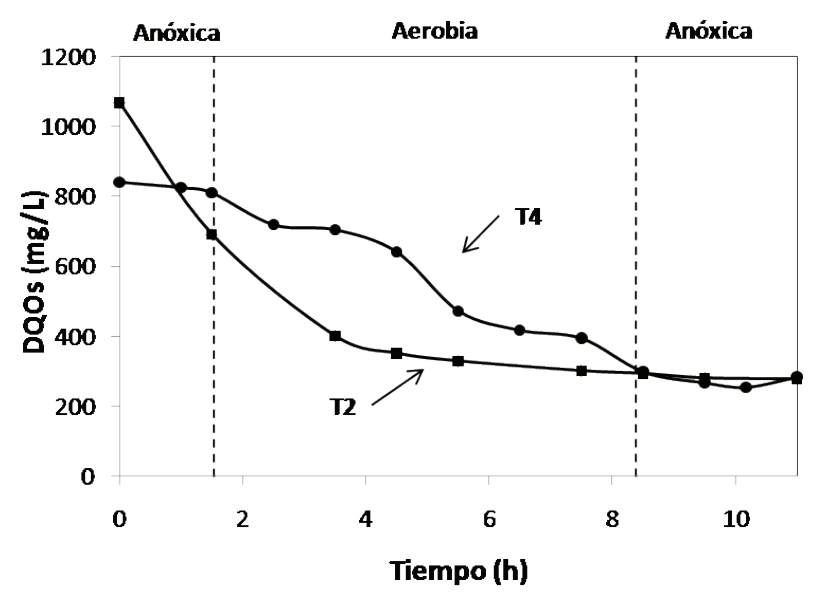

Figura 5. Evolución de la $\mathrm{DQO}_{\mathrm{S}}$ durante un ciclo de operación del SBR para los tratamientos T2 y T4

diciones anóxicas, el ORP decreció a valores negativos en todos los tratamientos aplicados. Los valores de ORP son principalmente dependientes de la concentración de OD en la etapa aerobia, están correlacionados con el logaritmo de la concentración del mismo en una relación lineal (Kishida et al., 2003).

De esta manera, el incremento en el ORP durante la fase aerobia fue debido al incremento del OD. Por otro lado, el potencial del óxido-reducción provee mucha mejor información sobre los procesos que ocurren en la fase anóxica que el oxígeno disuelto, esto debido a que cuando el OD disminuye a valores cercanos a cero, el ORP en el SBR se produce por el sistema $\mathrm{N}^{-N^{-}}{ }_{x}^{-}$(Akin y Ugurlu, 2005). El ORP y el OD suelen utilizarse para la optimización de la longitud de las fases, y de esta manera mejorar la eficiencia en los procesos de elimina- 
ción de nitrógeno, nitrificación y desnitrificación (Spagni et al., 2007).

Para el tratamiento T1, se observa que durante la primera hora y media del ciclo operacional no hubo variaciones significativas en las concentraciones de NTK y $\mathrm{N}-\mathrm{NH}_{4}{ }_{4}^{+}$, pero sí de $\mathrm{N}^{-\mathrm{NO}_{3}}{ }_{3}^{-}$producto del proceso de desnitrificación que ocurría en el reactor durante la etapa anóxica, el cual se confirma con la disminución de la concentración de nitratos, los valores negativos de ORP y la liberación de alcalinidad. Luego, en la fase aerobia se inicia el proceso de nitrificación, el cual se lleva a

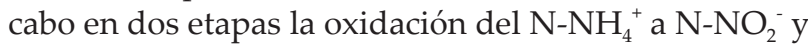
la oxidación del $\mathrm{N}^{-\mathrm{NO}_{2}}{ }^{-}$a N-NO${ }_{3}^{-}$en el cual la concentración de ${\mathrm{N}-\mathrm{NO}_{3}}_{3}^{-}$se incrementa constantemente a partir de la cuarta hora del ciclo.

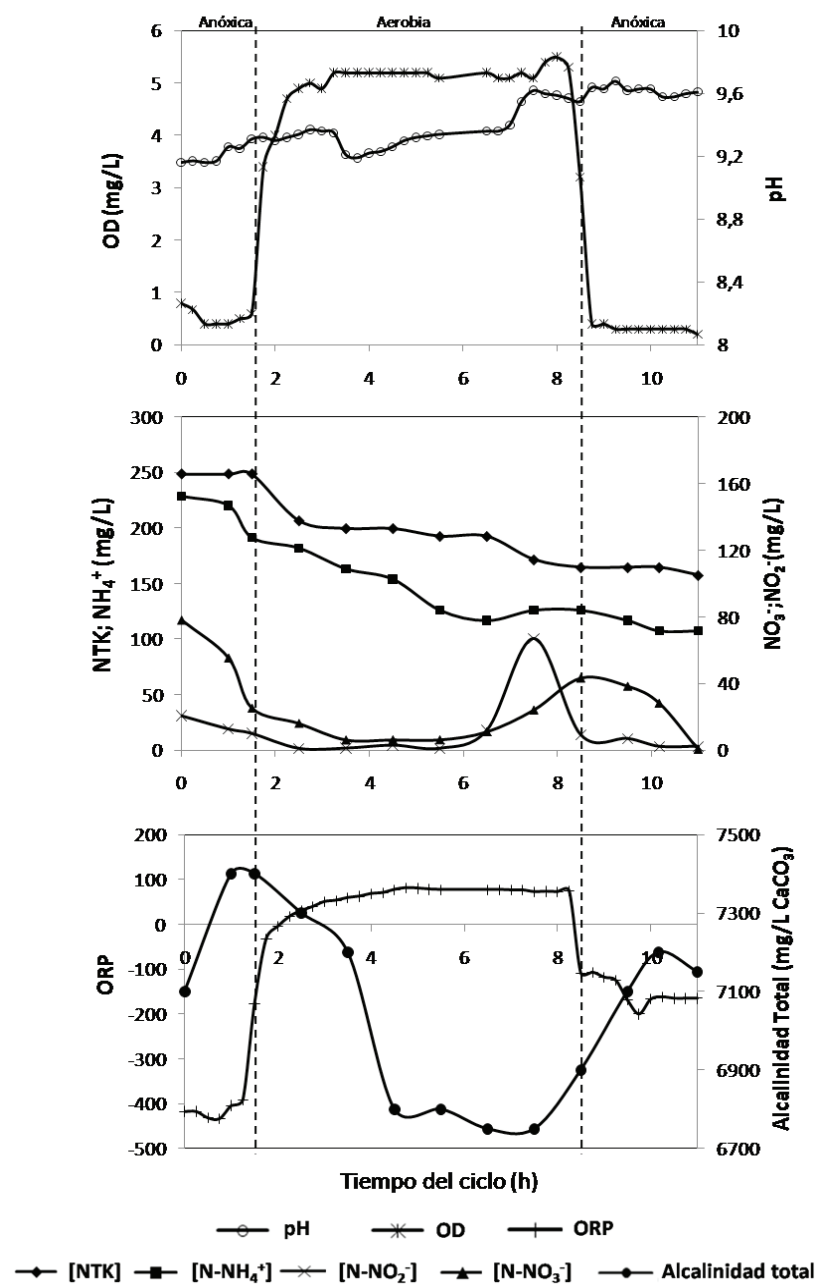

Figura 6. Perfil de las concentraciones de $\mathrm{NTK}, \mathrm{N}-\mathrm{NH}_{4}{ }^{+}$, $\mathrm{N}-\mathrm{NO}_{3}, \mathrm{~N}-\mathrm{NO}_{2}$, $\mathrm{pH}, \mathrm{OD}, \mathrm{ORP}$ y alcalinidad total durante el ciclo operacional de $11 \mathrm{~h}$ del SBR en el tratamiento T1
Para este tratamiento el proceso de nitrificación fue incompleto debido a que no todo el nitrógeno amoniacal fue transformado a nitratos, al final de la etapa aerobia se registraban valores de $107.3 \mathrm{mg} / \mathrm{L}$ de N-NH${ }_{4}{ }^{+}$. Se observa que durante la fase aerobia ocurre una disminución progresiva de las concentraciones de NTK y $\mathrm{N}-\mathrm{NH}_{4}{ }^{+}$producto del proceso de nitrificación. Al final de la etapa aerobia y comienzo de la segunda etapa anóxica de igual manera que en la primera, se produce una disminución de los nitratos debido al proceso de desnitrificación permitiendo obtener a la salida del tratamiento un efluente con concentraciones de $0.17 \mathrm{mg} / \mathrm{L}$ de $\mathrm{N}^{-\mathrm{NO}_{3}}{ }_{3}^{-}$y $2.23 \mathrm{mg} / \mathrm{L}$ de $\mathrm{N}-\mathrm{NO}_{2}{ }^{-}$.

Los valores de $\mathrm{pH}$ registrados en el ciclo operacional oscilan entre 9.2 y 9.6 unidades de $\mathrm{pH}$, con un aumento constante durante el ciclo operacional. El pH en la etapa aerobia se mantuvo entre 9.30 y 9.4 unidades. Estos valores se encuentran fuera del rango de $\mathrm{pH}$ óptimo para que ocurra la nitrificación, el cual está entre 7.5 y 8.6 unidades de $\mathrm{pH}$ (Louzeiro et al., 2002).

El comportamiento del $\mathrm{pH}$ difiere del reportado por Spagni et al. (2007); Han et al. (2007) y Li et al., (2008), quienes observaron un aumento del $\mathrm{pH}$ durante la etapa de desnitrificación y un descenso del mismo durante la fase de nitrificación, debido a la generación de iones hidronios que ocasionan este descenso. Por el contrario, el pH se incrementó continuamente y alcanzó un valor relativamente constante en la segunda etapa anóxica para el tratamiento T1.

La razón del inusual comportamiento del $\mathrm{pH}$ en el sistema SBR en estudio, es la capacidad de amortiguación o capacidad buffer que tienen las aguas residuales de tenerías que impiden las variaciones típicas del $\mathrm{pH}$ en los procesos que se llevan a cabo en el SBR (Garbagnati et al., 2005). Lee et al., 2001 encontraron un comportamiento similar en la fase aerobia y un fuerte incremento del pH en la fase anóxica debido al consumo de iones $\mathrm{H}^{+}$durante el consumo de fosfato anóxico en el reactor. Este consumo también se observa en la reacción estequiométrica de desnitrificación.

Con los valores de $\mathrm{pH}$ reportados se evita la inhibición de la nitrificación por ácido nitroso. Cuando el pH decrece, producto de la oxidación del amonio, la concentración de ácido nitroso libre se incrementa y junto a una concentración inicial de $\mathrm{N}^{-\mathrm{NH}_{4}}{ }^{+}$mayor a $500 \mathrm{mg} / \mathrm{L}$, puede ocurrir la inhibición de las Nitrosomonas y Nitrobacter (Obaja et al., 2003).

Respecto a las mediciones de la concentración de oxígeno disuelto en el reactor por carga secuencial, esta variable se mantuvo durante las fases anóxicas en valores menores a los $0.5 \mathrm{mg} / \mathrm{L}$ y durante la fase aerobia entre valores de 3.4 y $5.2 \mathrm{mg} / \mathrm{L}$ para el tratamiento $\mathrm{T} 1$, 
entre 2.4 y $6.0 \mathrm{mg} / \mathrm{L}$ para el tratamiento T2; 2.3 y 5.3 $\mathrm{mg} / \mathrm{L}$ para $\mathrm{T} 3$, y entre 1.5 y $5.9 \mathrm{mg} / \mathrm{L}$ para el tratamiento T4. La concentración de OD que se mide en el licor mezcla es la diferencia entre el oxígeno introducido por la aireación y el oxígeno al mismo tiempo consumido por los microorganismos. Dosta et al. (2008), reportaron altos valores de oxígeno disuelto $(\mathrm{OD} \geq 5 \mathrm{mg} / \mathrm{L})$ en un reactor por carga secuencial que trataba el efluente de una digestión anaeróbica mesofílica de aguas residuales de un matadero de cerdos.

Para todos los tratamientos se observó un incremento inicial en el oxígeno disuelto en la etapa aerobia, este incremento corresponde a la disminución de la actividad respiratoria bacteriana debido al agotamiento progresivo del sustrato (Spagni et al., 2007). Los valores medidos de OD limitan la ocurrencia del proceso de nitrificación-desnitrificación simultánea, el cual se ve inhibido a concentraciones de OD superiores de 2.5 $\mathrm{mg} / \mathrm{L}$ (Zeng et al., 2003), por lo que la eliminación de nitrógeno en todos los tratamientos aplicados ocurre por el proceso convencional de nitrificación en la fase aerobia y desnitrificación en la fase anóxica.

Estos niveles de oxígeno indican que el proceso de nitrificación se realiza, en su mayoría, por las bacterias nitrificantes autótrofas, ya que los altos niveles de OD favorecen el crecimiento de este tipo de bacterias, por encima de las bacterias heterotróficas nitrificantes, las cuales, pueden crecer mucho más rápido que las autotróficas a niveles muy bajos de OD hasta menores de 0.5 $\mathrm{mg} / \mathrm{L}$ (Zhao, 1999).

El oxígeno disuelto es la variable más importante en el proceso de nitrificación, en este estudio el suministro de flujo de aire fue constante durante la fase aerobia y la concentración de OD en el reactor cambiaba con la proporción de uso del OD. El máximo valor de OD disuelto corresponde al máximo valor de nitratos en el perfil de la fase aerobia y al mínimo valor de $\mathrm{N}_{-} \mathrm{NH}_{4}^{+} \mathrm{y}$ NTK (figura 7). Resultados similares fueron encontrados por Akin y Ugurlu, 2005; Han et al. (2007) y Dosta et al. (2008).

La curva de OD aumenta progresivamente en la fase aerobia a medida que va disminuyendo la curva de NTK y de $\mathrm{N}^{-\mathrm{NH}_{4}}{ }^{+}$, que indica que las bacterias quimilitótrofas llevan a cabo eficientemente los mecanismos de asimilación oxidativa donde se transforma el nitrógeno orgánico en nitrógeno amoniacal libre y este posteriormente se oxida a nitrito y a nitrato.

La curva de OD aumenta hasta estabilizarse en un valor de $5.7 \mathrm{mg} / \mathrm{L}$ de OD, punto en el cual se alcanza el mínimo valor de nitrógeno amoniacal en el ciclo operacional, posteriormente la curva de OD alcanza el valor máximo de $6.0 \mathrm{mg} / \mathrm{L}$, punto que corresponde al mínimo valor alcanzado de NTK. Luego el valor de OD disminuye a valores de $5.7 \mathrm{mg} / \mathrm{L}$ para luego caer bruscamente debido al comienzo de la fase anóxica. Este punto corresponde al máximo valor de la concentración de nitratos.

Leta et al. (2004), observaron un aumento en la eficiencia del proceso de nitrificación, al aumentar el OD. Las mayores eficiencias las reportaron a concentraciones superiores a $4 \mathrm{mg} / \mathrm{L}$. A $4.5 \mathrm{mg} / \mathrm{L}$ y $5.0 \mathrm{mg} / \mathrm{L}$ se obtuvieron eficiencias de $72 \%$ y $81 \%$, respectivamente y cuando la concentración fue de $6 \mathrm{mg} / \mathrm{L}$ se obtuvieron eficiencias de $93 \%$.

Para todos los tratamientos se observa una disminución de los nitratos en la fase anóxica posterior debido a la desnitrificación con una liberación de la alcalinidad y una disminución brusca del potencial de óxido reducción (ORP) producto del corte en la aireación, y por tanto, del suministro de oxígeno disuelto. En el proceso de

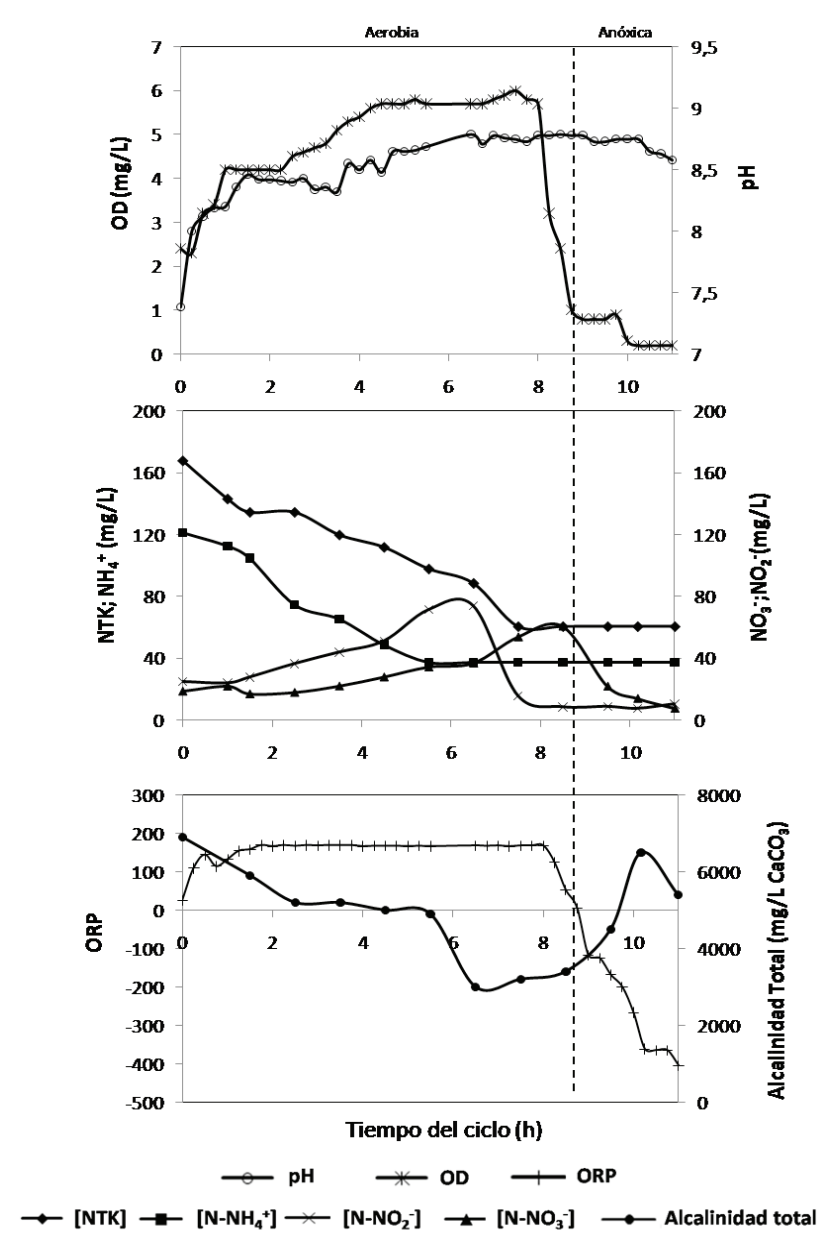

Figura 7. Perfil de las concentraciones de $\mathrm{NTK}, \mathrm{N}-\mathrm{NH}_{4}{ }^{+}, \mathrm{N}-\mathrm{NO}_{3}{ }^{-}$, $\mathrm{N}-\mathrm{NO}_{2}, \mathrm{pH}, \mathrm{OD}, \mathrm{ORP}$ y alcalinidad total durante un ciclo de 11 $h$ durante el tratamiento T2 en el SBR 
desnitrificación ocurren reacciones de reducción, las cuales solo proceden bajo condiciones anóxicas, condiciones en las cuales el ORP tiende a disminuir.

Normalmente, una fuente de carbono es necesaria para el proceso de post-desnitrificación, con una relación aproximada de $\mathrm{DQO} / \mathrm{NO}_{x}$ ' que oscila entre 5.0-6.0 (Li et al., 2007). Sin embargo, en esta investigación se encontró que la desnitrificación se llevó a cabo sin fuente de carbono externa.

$\mathrm{Al}$ final de la fase anóxica posterior, se alcanzaron valores de ORP de $-165,-405,-337$ y $-245 \mathrm{mV}$ para los tratamientos T1, T2, T3 y T4. Se observa que los tratamientos que alcanzaron menores valores ORP alcanzaron mayores eficiencias de desnitrificación durante la fase anóxica.

Akin y Ugurlu, 2005, demostraron que mientras más negativos eran los valores de ORP, mayores eran las tasas de desnitrificación y las tasas de liberación del

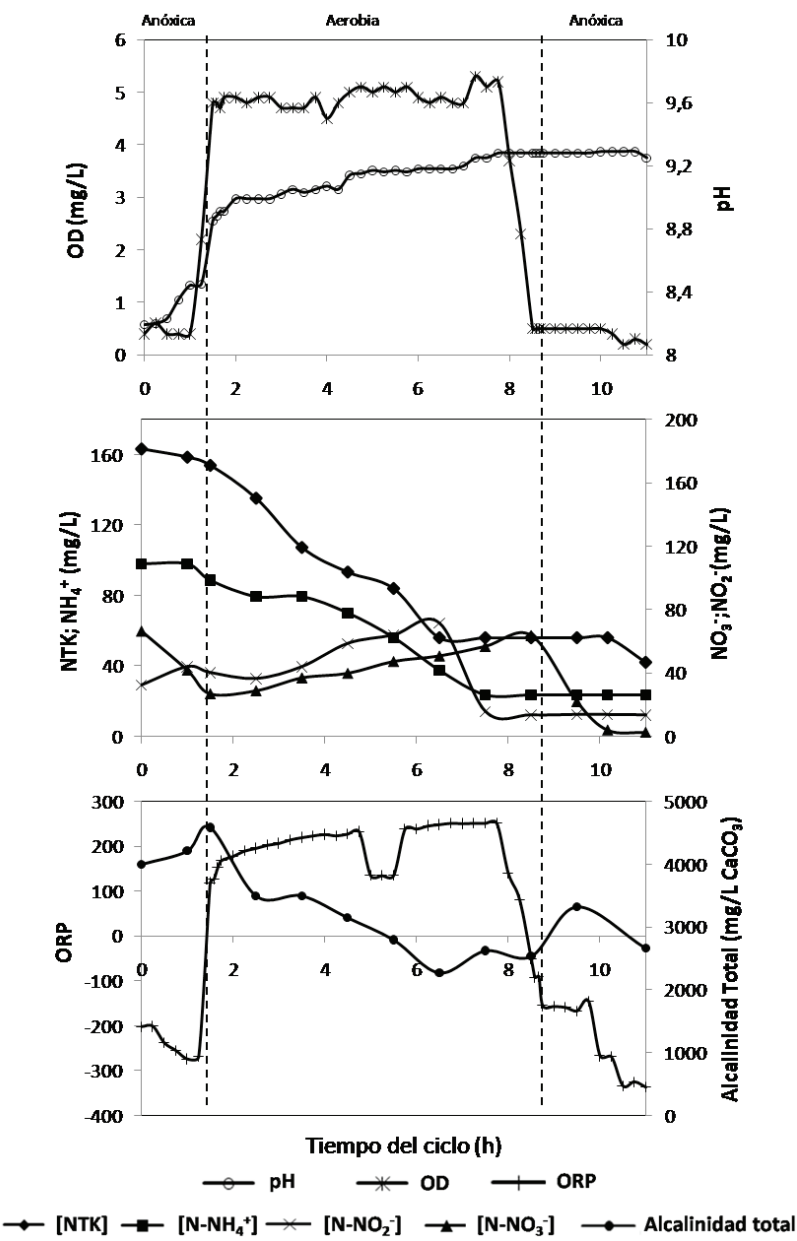

Figura 8. Perfil de las concentraciones de $\mathrm{NTK}, \mathrm{N}-\mathrm{NH}_{4}{ }^{+}, \mathrm{N}-\mathrm{NO}_{3}$, $\mathrm{N}-\mathrm{NO}_{2}, \mathrm{pH}, \mathrm{OD}, \mathrm{ORP}$ y alcalinidad total durante un ciclo de $11 \mathrm{~h}$ durante el tratamiento T3 en el SBR fosfato que se alcanzaban en el sistema de tratamiento. Cuando las condiciones eran aerobias, los valores de ORP se convertían a valores positivos, y mientras más positivos eran los valores registrados, mayores eran las tasas de consumo de fosfato.

El perfil de ORP es muy efectivo para el control de los procesos que ocurren en la fase anóxica. Cuando los nitratos se agotan en esta fase, un cambio en el perfil de ORP se observa, este punto se denomina rodilla del nitrato. Este punto indica el fin de la desnitrificación y la desaparición completa de los nitratos (Guo et al., 2006; Wu et. al., 2007; Hun et al., 2007; Qureshi et al., 2008; Yah y Hu, 2009). Sin embargo, algunas veces este punto no es fácil de detectar y aplicar en el funcionamiento práctico de los sistemas SBR. La precisión de las sondas de ORP se cuestiona debido a las incrustaciones en la sonda después de sumergirse en las aguas residuales por un periodo determinado (Li e Irvin, 2007).

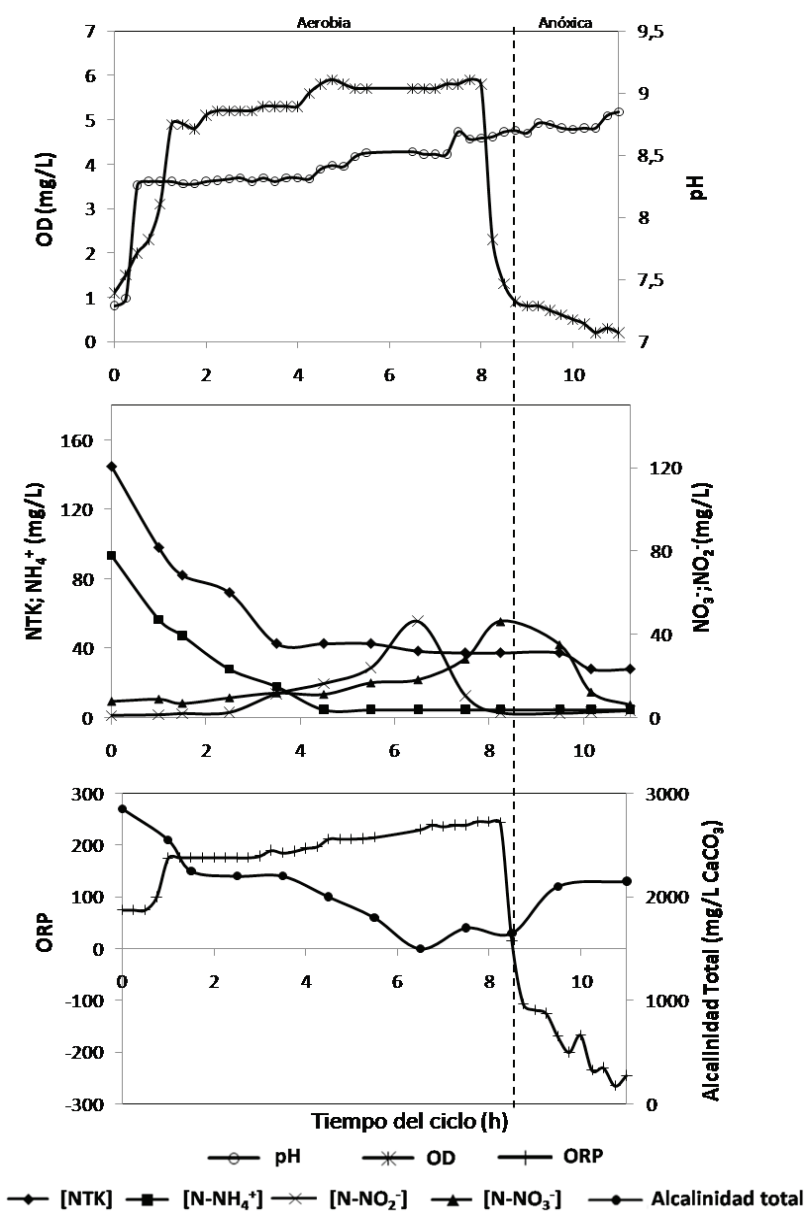

Figura 9. Perfil de las concentraciones de $\mathrm{NTK}, \mathrm{N}-\mathrm{NH}_{4}{ }^{+}, \mathrm{N}-\mathrm{NO}_{3}$, $\mathrm{N}-\mathrm{NO}_{2}, \mathrm{pH}, \mathrm{OD}, \mathrm{ORP}$ y alcalinidad total durante un ciclo de 11 h durante el tratamiento T4 en el SBR 
El pH y el ORP se han estudiado como indicadores para la nitrificación/desnitrificación. Sin embargo, se reportan resultados contradictorios; los puntos de control que indican el inicio/final de las fases de nitrificación/desnitrificación se detectaron en algunas pruebas (Akin y Ugurlu, 2005; Han et al., 2007; Spagni et al., 2007) pero no en otras ( $\mathrm{Hu}, 2005)$.

A pesar de que varios estudios han reportado que el $\mathrm{pH}$ es un buen indicador en el proceso de eliminación de nitrógeno, Hamamoto et al. (1997), encontraron que el $\mathrm{pH}$ se mantuvo estable, mientras que el ORP cambiaba significativamente a lo largo de los procesos aeróbicos/anóxicos. Resultados similares se encontraron en esta investigación.

De acuerdo con los resultados para la alcanilidad, esta presentó variaciones significativas durante el ciclo operacional durante cada tratamiento, por lo que puede ser utilizada como indicador de los procesos de nitrificación y desnitrificación en aguas residuales de tenerías.

En comparación con el $\mathrm{pH}$, la alcalinidad está directamente relacionada con la eliminación de nitrógeno, por lo que esta variable puede utilizarse como parámetro de control en este proceso con más confianza que el $\mathrm{pH}$. Aunque la vinculación de la alcalinidad y la nitrificación/ desnitrificación se conoce desde hace décadas sobre una base científica, existe muy poca información disponible acerca de la relación entre la alcalinidad y la concentración de nitrógeno de efluentes (Li e Irvin, 2007)

Para todos los tratamientos se observó un consumo de la alcalinidad durante la nitrificación y un aumento durante la desnitrificación.

\section{Conclusiones}

Los cuatro tratamientos aplicados al agua residual industrial de la tenería en el reactor por carga secuencial (SBR) permitieron la remoción de nitrógeno y materia orgánica, debido a la flexibilidad del reactor para adaptarse a distintas condiciones de operación.

El pH no puede utilizarse como un parámetro de control y operación en la remoción de nitrógeno en un sistema SBR para el efluente de tenería en estudio debido a su capacidad de amortiguación que impide variaciones significativas en el $\mathrm{pH}$.

Los resultados obtenidos muestran que la alcalinidad total y el ORP son parámetros que pueden utilizarse para en el monitoreo y control en la remoción biológica nitrógeno durante el tratamiento de efluentes de tenerías, debido a que se observó para todos los tratamientos un consumo de la alcalinidad total durante la nitrificación y un aumento de la misma durante la desnitrificación acompañado de una disminución en los valores de ORP durante las fases anóxicas. Además, las fases de cada ciclo operacional pueden ser identificadas claramente de acuerdo a los perfiles de estas variables.

\section{Referencias}

Akin B., Ugurlu A. Monitoring and Control of Biological Nutrient Removal in a Sequencing Batch Reactor. Process Biochemistry, volumen 40, 2005: 2873-2878.

American Public Health Association (APHA-AWWA-WEF). On: CLESCERI, L.; GREENBERG, A.; EATON, A. (Ed). Standard Methods for the Examination of Water and Wastewater. $20^{\text {th }}$ edition. American Public Health Association, Washintong D.C., 1998, pp. I-48

Carucci A., Majone M., Ramadori R., Rosetti S. Biological Phosphorus Removal with Different Organic Substrates in an Anaerobic/Aerobic Sequencing Batch Reactor. Water Science Technology, volumen 35, 1997: 161-187.

Caruccí A., Chiavola A., Majone M., Rolle, E. Treatment of Tannery Wastewater in a Sequencing Batch Reactor. Water Science and Technology, volumen 40, 1999: 253-259.

DiIaconi C., Lopez A., Ramadorai R., Di Pinto A., Passino R. Combined Chemical and Biological Degradation of Tannery Wastewater by a Periodic Submerged Filter (SBBR). Water Res., volumen 36, 2002: 2205-2214.

Dosta J., Rovira J., Galí A., Macé S., Mata-Alvarez J. Integration of a Coagulation/Flocculation Step in a Biological Sequencing Batch Reactor for COD and Nitrogen Removal of Supernatant of Anaerobically Digested Piggery Wastewater. Bioresource Technology, volumen 13, 2008: 5722-5730.

Durai G., Rajasimman M. Biological Treatment of Tannery Wastewater - A Review. Journal of Environmental Science and Tecnology, volumen 4 (número 1), 2011: 1-17.

Farabegoli G., Caruccí A., Majone M., Rolle E. Biological Treatment of Tannery Wastewater in the Presence of Chromium. Journal of Environmental Management, volumen 71, 2004: 345-349.

Gaceta Oficial de la República de Venezuela. Decreto Núm. 883. Normas para la clasificación y el control de la calidad de los cuerpos de agua y vertidos o efluentes líquidos. Gaceta Oficial Núm. 5021, Venezuela, 1995, p. 17.

Garbagnati M., González S., Antón, R., Mallea M. Características físico-químicas, capacidad buffer y establecimiento de la línea base ambiental del Río Grande, San Luis, Argentina. Ecología Austral, volumen 15, 2005: 59-71.

Ganesh R., Balaji G., Ramanujam A. Biodegradation of Tannery Wastewater Using Sequencing Batch Reactor- Respirometric Assessment. Bioresource Technology, volumen 97, 2006: 1815-1821.

Guo J., Yang Q., Peng Y., Yang A. Wang S. Biological Nitrogen Removal with Real-Time Control Using Step-Feed SBR Technology. Enzyme and Microbial Technology, volumen 40 (número 6), 2007: 1564-1569. 
Hamamoto Y., Tabata S., Okubo Y. Development of the Intermittent Cyclic Process for Simultaneous Nitrogen and Phosphorous Removal. Water Science Technology, volumen 35 (número 1), 1997: 145-152.

Han Z., Wu W., Chen Y., Zhu J. Characteristics of a Twice-Fed Sequencing Batch Reactor Treating Swine Wastewater Under Control of Aeration Intensity. Journal of Environmental Science and Health, volumen 42, 2007: 361-370.

Hermida V., Galisteo S., Vinas S. Evaluación respirométrica de la biodegrabilidad aeróbica de un efluente de curtiembre. Estrucplan [en línea][fecha de consulta: 25 de febrero de 2000], Disponible en: www.estrucplan.com.mx.

Hu Z., Ferraira R., Ericson J., Mackay A., Smets B. Biomass Characteristics in Three Sequencing Batch Reactors Treating a Wastewater Containing Synthetic Organic Chemicals. Water Research, volumen 39, 2005: 710-720.

Hun S., Woo M., Sung D., Moon J. Monitoring of Sequencing Batch Reactor for Nitrogen and Phosphorus Removal Using Neural Networks. Journal Biochemical Engineering, volumen 35, 2007: 365-370.

Karahan Ö., Dogruel S., Dulekgurgen E., Orhon D. COD Fractionation of Tannery Wastewaters -Particle Size Distribution, Biodegradability and Modeling. Water Research, volumen 42, 2008:1083-1092.

Kargi F., Uygur A. Nutrient Removal Performance of a Five-Step Sequencing Batch Reactors a Function of Wastewater Composition. Process Biochemistry, volumen 38, 2003:1039-1045.

Kishida N., Kim J., Chen M., Sasaki H., Sudo R. Effectiviness of Oxidation-Reduction Potential and $\mathrm{pH}$ as Monitoring and Control Parameters for Nitrogen Removal in Swine Wastewater by Sequencing Batch Reactors. Journal of Bioscience and Bioengineering, volumen 96 (número 3), 2003: 285-90.

Lee D., Jeon C., Park J. Remoción biológica de nitrógeno con consumo mejorado de fosfato en un reactor por carga secuencial usando un sistema de lodos simple. Water Research, volumen 35 (número 16), 2001: 3968-3976.

Lefebvre O., Vasudevan N., Torrijos M., Thanasekaran K, Moletta R. Halophilic Biological Treatment of Tannery Soak Liquor in a Sequencing Batch Reactor. Water Research, volumen 39, 2005: 1471-1480.

Leta S., Assefa F., Gumaelis L., Dalhammar G. Biological Nitrogen and Organic Removal Fromm Tannery Wastewater in Pilot Plant Operations in Ethiopia. Applied microbial Biotechnology, volumen 66, 2004: 333-3339.

Li B., Irvin S. The Comparison of Alkalinity and ORP as Indicators for Nitrification and Denitrification in a Sequencing Batch Reactor (SBR). Biochemical Engineering Journal, volumen 34, 2007: 248-255.

Li J., Healy M., Zhan X., Norton D., Rodgers M. Effect of Aeration Rate on Nutrient Removal from Slaughterhouse Wastewater in Intermittently Aerated Sequencing Batch Reactors. Water Air Soil Pollution, volumen 192, 2008: 251-261.
Louzeiro N., Mavinic D., Oldham W., Meisen A., Gardner I. Methanol Induced Biological Nutrient Removal Kinetics in a Full Scale Sequenting Batch Reactor. Water Research, volumen 36, 2002: 2721-2732.

Mace S., Mata-Álvarez J. Utilization of SBR Technology for Wastewater Treatment: an Overview. Industrial \& Engineering Chemistry Research, volumen 41, 2002: 5539-5553.

Obaja D., Macé S., Costa J., Sans C., Mata-Alvarez, J. Nitrification, Denitrification and Biological Phosphorus Removal in Piggery Wastewater Using a Sequencing Bacth Reactor. Bioresource Technology, volumen 87, 2003: 103-111.

Orhon D., Ubay E. COD Fractionation in Wastewater Characterization-The State of the Art. Journal of Chemical Technology and Biotechnology, volumen 68 (número 3), 1997: 283-293.

Orhon D., Sözen S., Ubay E. Experimental Basis for the Hydrolysis of Slowly Biodegradable Substrate in Different Wastewaters. Water Science and Technology, volumen 39 (número 1), 1999a: 87-95.

Orhon D., Karahan O., Sozen S. The Effect of Residual Microbial Products on the Experimental Assessment of the Particulate Inert COD in Wastewaters. Waters Research, volumen 30, 1999b: 3191-3203.

Orhon D., Ates A., Sözen S. Experimental Evaluation of the Nitrification Kinetics for Tannery Wastewaters. Water $S A$, volumen 26 (número 1), 2000: 43-50.

Qureshi A., Lo V., Ping L., Mavinic D. Real-Time Treatment of Dairy Manure: Implications of Oxidation Reduction Potential Regimes to Nutrient Management Strategies. Bioresource Technology, volumen 99 (número 5), 2008: 1169-1176.

Spagni A., Buday J., Ratini P., Bortone G. Experimental Considerations on Monitoring ORP, $\mathrm{pH}$, Conductivity and Dissolved Oxygen in Nitrogen and Phosphorus Biological Removal Processes. Water Science and Technology, volumen 43 (número 11), 2001: 197-204.

Spagni A., Lavagnolo C., Scarpa C., Vendrame P., Rizzo A., Lucarrini L. Nitrogen Removal Optimization in a Sequencing Batch Reactor Treating Sanitary Landfill Leachate. Journal of Environmental Science and Health, volumen 42, 2007: 757-765.

Sivaparksam S., Surianarayanan M., Sekar S., Rajakumar S. Biological Treatment of Tannery Wastewater by Using Salt-Tolerant Bacterial Strains. Microbial Cell Factories, volumen 7, 2008:1-15.

Szpyrkowics L., Kaul S. Biochemical Removal of Nitrogen from Tannery Wastewater: Performance and Stability of a Full Scale Plant. Journal of Chemical Technology and Biothecnology, volumen 79, 2004: 879-888.

Tanwar P., Nandy T., Ukey P., Maneka P. Correlating On-Line Monitoring Parameters, $\mathrm{pH}, \mathrm{DO}$, and ORP with Nutrient Removal in a Intermittent Cyclic Process Bioreactor System. Bioresource Technology, volumen 99, 2008: 7630-7635.

U.S. Environmental Protection Agency (EPA). Wastewater Technology Fact Sheet Sequencing Batch Reactors, EPA 832-F-99073, Washington D.C., 1999. 
Vidal G., Nieto J., Cooman K., Gajardo M., Bornhardt C. Unhairing Effluents Treated by an Activated Sludge System. Journal of Hazardous Materials B, volumen 112, 2004: 143-149.

Wu C., Chen Z., Liu X., Peng Y. Nitrification-Denitrification Via Nitrite in SBR Using Real-Time Control Strategy when Treating Domestic Wastewater. Biochemical Engineering Journal, volumen 36 (número 2), 2007: 87-92.

Yan J., Hu Y. Comparison of Partial Nitrification to Nitrite for Ammonium-Rich Organic Wastewater in Sequencing Batch Reactors and Continuous Stirred-Tank Reactor at LaboratoryScale. Water Science and Technology, volumen 60 (número 11), 2009: 2861-2868.

Zeng R., Lemaire R., Yuan Z., Keller J. Simultaneous Nitrification, Denitrification and Phosphorus Removal in a Lab-Scale Sequencing Batch Reactor. Biotechnology and Bioengineering, volumen 84 (número 2), 2003: 171-177.

Zhao W., Mavinic S., Oldham K., Kloch A. Controling Factors for Simultaneous Nitification and Denitrification in a Two Stage
Intermittent Aereation Process Treating Domestic Sewage. Water Research, volumen 33 (número 4), 1999: 961-70.

\section{Este artículo se cita: \\ Citación estilo Chicago \\ Carrasquero-Ferrer, Sedolfo José, María Carolina Pire-Sierra, Nan- cy Coromoto Rincón-Lizardo, Altamira Rosa Díaz-Montiel. Moni- toreo de la remoción biológica de nitrógeno en efluentes de tenerías usando un reactor por carga secuencial. Ingeniería Inves- tigación y Tecnología, XV, 02 (2014): 287-298.}

\section{Citación estilo ISO 690}

Carrasquero-Ferrer S.C., Pire-Sierra M.C., Rincón-Lizardo N.C, Díaz-Montiel A.R. Monitoreo de la remoción biológica de nitrógeno en efluentes de tenerías usando un reactor por carga secuencial. Ingeniería Investigación y Tecnología, volumen XV (número 2), abril-julio 2014: 287-298.

\section{Semblanza de los autores}

Sedolfo José Carrasquero-Ferrer. Es ingeniero químico por la Universidad del Zulia (2007), obtuvo el grado de magister scientiarum en ingeniería ambiental por la Universidad del Zulia en 2011. Actualmente se desempeña como profesor e investigador a dedicación exclusiva del Departamento de Ingeniería Sanitaria y del programa de postgrado de ciencias del ambiente de la Universidad del Zulia, Maracaibo, Venezuela.

María Carolina Pire-Sierra. Es ingeniero químico por la Universidad Nacional Experimental Politécnica (2000) en Barquisimeto, Venezuela. Obtuvo el grado de magister scientiarum en ingeniería ambiental por la Universidad del Zulia (2009), así como también el título de doctora en ingeniería ambiental (2012). Es profesora e investigadora a tiempo completo del programa de ingeniería agroindustrial de la Universidad Centroccidental Lisandro Alvarado (UCLA), Barquisimeto, Venezuela.

Nancy Coromoto Rincón-Lizardo. Es ingeniero civil por la Universidad del Zulia (1991), donde también obtuvo el grado de magister scientiarum en ingeniería ambiental en 1996. Realizó estudios doctorales en el Instituto Nacional de Ciencias Aplicadas en Toulouse, Francia, donde obtuvo el grado de doctora en ingeniería del proceso y medio ambiente (2002). Además realizó estudios posdoctorales en ingeniería ambiental en la Universidad de Barcelona, España (2005). Es profesora investigadora a dedicación exclusiva del Departamento de Ingeniería Sanitaria, del programa de postgrado de ciencias del ambiente y del programa de doctorado en ingeniería ambiental de la Universidad del Zulia, Maracaibo, Venezuela.

Altamira Rosa Díaz-Montiel. Es ingeniero civil por la Universidad del Zulia (1991), donde también obtuvo el grado de magister scientiarum en ingeniería ambiental en 1996. Realizó estudios doctorales en el Instituto Nacional de Ciencias Aplicadas en Toulouse, Francia, donde obtuvo el grado de doctora en ingeniería de procesos y medio ambiente (2002). Es profesora e investigadora a dedicación exclusiva del Departamento de Ingeniería Sanitaria, coordinadora del programa de postgrado de ciencias del ambiente de la Universidad del Zulia, Maracaibo, Venezuela. 